\title{
Cell Proliferation in Adult Hippocampus is Decreased by Inescapable Stress: Reversal by Fluoxetine Treatment
}

\author{
Jessica E Malberg' and Ronald S Duman*,2 \\ 'Wyeth Neuroscience, Princeton, NJ, USA; '2 Laboratory of Molecular Psychiatry, Center for Genes and Behavior, Departments of Psychiatry and \\ Pharmacology, Yale University School of Medicine, New Haven, CT, USA
}

\begin{abstract}
Adult hippocampal neurogenesis has been demonstrated in several species and is regulated by both environmental and pharmacological stimuli. The present study seeks to determine whether hippocampal proliferation and neurogenesis are altered in adult animals exposed to inescapable shock (IS) in the learned helplessness model of depression. We report that exposure to avoidance testing, regardless of pre-exposure to IS, decreases cell proliferation in the hippocampus, extending previous studies demonstrating downregulation of neurogenesis by exposure to acute stressors. In addition, when the analysis was conducted 9 days after exposure to IS we observed a significant decrease in cell proliferation compared to nonshocked animals. Administration of fluoxetine, a serotonin selective reuptake inhibitor, from days 2-8 blocked the downregulation of cell proliferation resulting from IS. Fluoxetine treatment also reversed the deficit in escape latency observed in animals exposed to IS. Finally, at the 9 day time point, there was no significant difference in blood levels of corticosterone between nonshocked and IS exposed animals, indicating that the decreased cell proliferation that is observed is not due to increased levels of this adrenal steroid. These findings demonstrate that exposure to IS, which results in a state of behavioral despair, decreases hippocampal cell proliferation and that this effect can be reversed by fluoxetine treatment.

Neuropsychopharmacology (2003) 28, I 562 - I57I, advance online publication, 02 July 2003; doi: I 0. I 038/sj.npp. I 300234
\end{abstract}

Keywords: neurogenesis; antidepressant; learned helplessness; depression; corticosterone; footshock

\section{INTRODUCTION}

Adult hippocampal neurogenesis has been demonstrated in a variety of different species, and is regulated by environmental and pharmacological variables (Gage, 2000; Gould et al, 2000; Duman et al, 2001; Kempermann, 2002). For example, hippocampal neurogenesis in rat and nonhuman primates is increased by exercise, enriched environment, and hippocampal-dependent learning, but decreased by exposure to stress or administration of adrenal glucocorticoids. The proliferating cells in the subgranular zone (SGZ) of the hippocampus give rise to mature neurons that migrate into the granule cell layer (Hastings and Gould, 1999). Moreover, these new neurons differentiate and appear to have morphological and physiological characteristics that are similar to adult granule cells (van Praag et al, 2002).

A role for neurogenesis in mood disorders such as depression and post-traumatic stress disorder (PTSD) has

\footnotetext{
*Correspondence: Dr RS Duman, 34 Park St., New Haven, CT 06508, USA, Tel: + I 203974 7726; Fax: + I 203974 7724;

E-mail: ronald.duman@yale.edu

Received 04 December 2002; revised 19 April 2003; accepted 12 May 2003

Online publication: 15 May 2003 at http://www.acnp.org/citations/ Npp05 I502446/default.pdf
}

been suggested based on preclinical and clinical studies of stress and antidepressant treatment. Exposure of nonhuman primates to intruder or psychosocial stress decreases proliferation in adult hippocampus (Gould et al, 1997, 1998), and exposure of rodents to predator odor stress produces a similar decrease in cell proliferation (Tanapat et al, 2001). In contrast, antidepressant treatment increases adult proliferation and neurogenesis in the hippocampus, and the time course for this effect (ie chronic, but not acute treatment) is consistent with the time course for the therapeutic action of antidepressants (Malberg et al, 2000; Madsen et al, 2000; Manev et al, 2001). In addition, administration of an atypical antidepressant is reported to block the downregulation of neurogenesis resulting from intruder stress (Czeh et al, 2001). Repeated stress also results in atrophy of CA3 pyramidal neurons in the hippocampus (Magarinos et al, 1996; McEwen, 1999), which is reversed by the atypical antidepressant tianeptine (Watanabe et al, 1992).

The potential clinical relevance of these experimental findings is supported by brain imaging studies demonstrating that the volume of the hippocampus is decreased in patients with major depression or PTSD (Bremner et al, 1995, 2000; Sheline et al, 1996). One study has reported that the magnitude of the reduction is correlated with the length of depressive illness, indicating that the change in 
hippocampal volume is caused by depression (Sheline et al, 1999). Gilbertson et al (2002) recently studied hippocampal volume in PTSD patients and their identical twins. The PTSD patients had a smaller hippocampal volume, which was negatively correlated with symptom severity. However, the unaffected identical twin also had reduced hippocampal volume, suggesting that the reduced volume produces a state of vulnerability for this stress-related disorder. The relationship between hippocampal volume and illness is still being investigated. It is not known if the reduced volume results from a decrease in the number of granule cells or other cell types or atrophy of hippocampal neurons. Additional imaging and post-mortem studies will be needed to address these questions, but the reduced hippocampal volume is consistent with the possibility that decreased neurogenesis and/or neuronal atrophy may occur in depressed patients.

The current series of experiments was designed to extend the preclinical studies of stress, antidepressants, and adult neurogenesis to the learned helplessness paradigm. In this model, exposure to inescapable stress, in this case footshock, results in an inability to escape shock in a subsequent avoidance test (AT) (Willner, 1984; Thiebot et al, 1992; Cryan et al, 2002). This paradigm has been used for both a screen for putative antidepressant drugs and as an animal model for depression. Its pharmacological validity comes from the findings that the escape deficit produced by exposure to inescapable shock (IS) can be reversed by subchronic, but not acute antidepressant treatment. Although it is difficult to define depression in a laboratory animal, exposure to IS results in many changes that have been compared to depressive symptoms in patients, including decreased motor activity, loss of appetite and weight, decreased self-stimulation, and deficits in immunosuppression (Thiebot et al, 1992). There are some drawbacks to the test, however, which should be mentioned, such as the high level of aversive shock needed to produce helplessness, variability between laboratories, and the large number of key variables that can affect the outcome of the test (Vollmayr and Henn, 2001; Cryan et al, 2002; Porsolt, 2000). Nonetheless, learned helplessness, along with the forced swim test, is an accepted and widely studied behavioral model of depression (Cryan et al, 2002).

In these experiments, cell proliferation was measured by incorporation of 5-bromo-2-deoxyuridine (BrdU), a thymidine analog that labels dividing cells in the S-phase (Takahashi et al, 1992). We report that exposure to inescapable foot-shock decreases hippocampal cell proliferation, and that fluoxetine treatment blocks this effect.

\section{METHODS}

\section{Animals}

Adult male Sprague-Dawley rats (175-225 g) (Harlan, Indianapolis, IN) were used in all experiments. All animal treatments and maintenance of the rat colony were in accordance with Guide for the Care and Use of Laboratory Animals and were approved by the Yale University Animal Care and Use Committee. Animals were group-housed $(12 \mathrm{~h}$ light/dark cycle) with ad libitum access to food and water.
Animals received at least 5 days of habituation to the rat colony before testing.

\section{Apparatus}

All behavioral tests were performed with the Gemini ${ }^{\mathrm{m}}$ active avoidance system (San Diego Instruments, San Diego, CA). The system is an enclosed shuttle box divided into two identical compartments separated by a retractable door. A grid floor delivers a scrambled shock to either side of the shuttle box. The latency to escape to the unshocked side is recorded by the Gemini system in all trials.

\section{Learned Helplessness Paradigm}

In this paradigm, an animal is initially exposed to IS on one side of the shuttle box. When the animal is later given shock from which it can escape, the animal fails to acquire the escape response. This escape deficit, termed learned helplessness, is reversed by chronic antidepressant treatment (Cryan et al, 2002).

The learned helplessness paradigm used in the present study was divided into three components: (1) IS training to induce learned helplessness; (2) postshock avoidance testing to confirm helplessness in shocked animals; and (3) a two-way conditioned AT.

On Day 1, all rats in the IS group were given 60 inescapable foot-shocks accompanied by a tone $(0.8 \mathrm{~mA}$ shock, $15 \mathrm{~s}$ duration shock + tone, ITI interval $=45 \mathrm{~s}$ average, range from 40 to $50 \mathrm{~s}$ ). A control group of animals who were not shocked (NS) were placed in the shuttle box for $1 \mathrm{~h}$ but did not receive any shock.

On Day 2, a two-way conditioned AT was performed on the IS group as a 'postshock test'. It has been demonstrated in our laboratory (Shirayama et al, 2002) that $25-30 \%$ of normal animals exposed to IS do not show the predicted escape deficit. This produces false positive results that could be misinterpreted as an antidepressant effect. To control for this, the postshock test was used to insure that the animals used in the rest of the experiment were truly 'helpless' before starting antidepressant treatments. The postshock test consisted of 30 trials. Animals were presented with a 3-s tone followed by an additional $3 \mathrm{~s}$ of tone + shock $(0.8 \mathrm{~mA}$, ITI interval $=30 \mathrm{~s}$ average, $25-35 \mathrm{~s}$ range). The gate separating the two sides of the shuttle box opened with the onset of the tone. When the rats crossed into the unshocked side of the box, the gate was closed and the tone was terminated. If the rat failed to escape, the shock was terminated at the end of the trial and the gate was closed. Rats with $>5$ escape failures in the last 10 trials were scored as having reached the helpless criterion and were used for experiments. NS rats were placed in the shuttle box for 20 min on Day 2.

On Day 9, animals were given a two-way conditioned AT. This time point was chosen because it allowed for 7 days (Days 2-8) of antidepressant treatment after the IS and postshock testing. The conditioned AT consisted of 30 trials. Animals were presented with a $3 \mathrm{~s}$ tone followed by $30 \mathrm{~s}$ tone + shock $(0.8 \mathrm{~mA}$, ITI interval $=30 \mathrm{~s}$ average, $25-35$ range). The door between the two sides of the shuttle box opened with the onset of the tone and remained open until the animal crossed to the unshocked side. If the rat failed to 
escape, the shock was terminated at the end of the trial and the gate was closed.

Two paradigms were used to determine the effect of learned helplessness on cell proliferation and escape latency (see Figure 1 for experimental design).

Experiment 1: IS and NS animals were treated on Days 1 and 2 as described above. On Day 9, all of the IS and half of the NS rats were given the AT (these two groups were then referred to at the IS/AT and NS/AT groups). At $5 \mathrm{~min}$ after they were removed from the testing shuttle box, the IS/AT and NS/AT groups were injected with BrdU. The other half of the NS group was not tested, but was injected with BrdU only.

Experiment 2: IS and NS animals were treated as above on Days 1 and 2. On Day 2, the IS animals were divided into two groups. One group (IS + FLX group) was given fluoxetine $(10 \mathrm{mg} / \mathrm{kg}$, b.i.d., received from Eli Lilly) on Days 2-8 and the other group (IS group) was given a saline injection on Days 2-8. The dose of fluoxetine was chosen based on pilot studies in the laboratory as the behaviorally effective dose in this paradigm. The NS group was given a saline injection $(1 \mathrm{ml} / \mathrm{kg})$ on Days $2-8$. On Day 9 , half of the animals in each treatment group (NS, IS, IS + FLX) were given a BrdU injection. The other half of the animals in each group was given the two-way conditioned AT (NS/AT, IS/ AT, IS + FLX/AT). All animals received either a BrdU injection or the AT on Day 9, but not both.

\section{Escapable Shock Paradigm}

On Day 1, animals were given a 30-trial two-way AT. This test was identical to the one used on Day 9 in the previous experiments (see above). In this way, animals were exposed to escapable shock (ES) on Day 1. On Day 2, animals were given the 30-trial postshock test as described above to insure that no animals had become spontaneously helpless. NS animals were exposed to the box only on Days 1 and 2. All animals in both groups were injected with saline on Days 2-8 and injected with BrdU and killed on Day 9.

\section{BrdU Labeling and Analysis}

For analysis of BrdU-positive cells, rats were administered BrdU ( $100 \mathrm{mg} / \mathrm{kg}$, i.p.; Sigma) as described above. At $2 \mathrm{~h}$ after the BrdU injection, all rats were killed and transcardially perfused ( $0.1 \mathrm{M}$ cold PBS for $5 \mathrm{~min}$ followed by $4 \%$ cold paraformaldehyde for $15 \mathrm{~min}$ ). This 2 -h time point was chosen because it represents one cell cycle of labeling (Cameron and McKay, 2001). For determination of cell survival and phenotype, a separate group of IS and NS rats were injected with BrdU on Day 9 and killed 28 days later.

Serial sections of the brains $(35 \mu \mathrm{m})$ were cut through the entire hippocampus on a freezing microtome. The sections were slide-mounted and boiled in citric acid followed by treatment with $0.1 \%$ trypsin in Tris/CaCl. The sections were

\begin{tabular}{l|l|c}
\multicolumn{1}{c|}{ Day 1 } & \multicolumn{1}{|c}{ Day2 } & Day 9 \\
\hline Inescapable shock (IS) & Post-shock test & $\begin{array}{c}\text { IS rats given avoidance test (IS/AT) } \\
\text { then injected with BrdU }\end{array}$ \\
OR & OR & $\begin{array}{c}\text { Half the NS rats given avoidance test (NS/AT) } \\
\text { then injected with BrdU }\end{array}$ \\
$\begin{array}{l}\text { No shock (NS): } \\
\text { exposure to shuttle } \\
\text { box only }\end{array}$ & $\begin{array}{l}\text { NS: shuttle box } \\
\text { only }\end{array}$ & $\begin{array}{l}\text { Half the NS rats injected with BrdU only (NS) } \\
\end{array}$
\end{tabular}

\begin{tabular}{|c|c|c|c|}
\hline Day 1 & Day2 & Days 2-8 & Day 9 \\
\hline Inescapable shock (IS) & Post-shock test & $\begin{array}{l}\text { After PS test, IS rats } \\
\text { divided into } 2 \text { treatment } \\
\text { groups: }\end{array}$ & $\begin{array}{l}\text { Half the rats in all } 3 \text { treatment } \\
\text { groups given avoidance test } \\
\text { (IS/AT, IS+FLX/AT, NS/AT) }\end{array}$ \\
\hline & & $\begin{array}{l}\text { IS: } 1 \mathrm{ml} / \mathrm{kg} \mathrm{SAL} \\
\text { IS+FLX: } 10 \mathrm{mg} / \mathrm{kg} \text { b.i.d }\end{array}$ & $\begin{array}{l}\text { Half the rats in all } 3 \text { treatment } \\
\text { groups injected with BrdU only } \\
\text { (IS, IS+FLX, NS) }\end{array}$ \\
\hline $\begin{array}{l}\text { No shock(NS): } \\
\text { exposure to shuttle } \\
\text { box only }\end{array}$ & $\begin{array}{l}\text { NS: shuttle box } \\
\text { only }\end{array}$ & NS: $1 \mathrm{ml} / \mathrm{kg} \mathrm{SAL}$ & $\begin{array}{l}\text { Animals are either used for } \\
\text { behavior or cell proliferation, } \\
\text { but not both }\end{array}$ \\
\hline
\end{tabular}

Figure I Experimental paradigms (a) experiment I: Rats were given IS or NS treatment and the postshock (PS) test. On Day 9, all of the IS and half of the NS rats were given the AT (IS/AT and NS/AT) followed by a BrdU injection. The other half of the NS group was not tested but was injected with BrdU only. (b) experiment 2: After IS and the PS test, and FLX or SAL treatment, all treatment groups were divided in half on Day 9 . Half of the animals in each treatment group was given BrdU injections and killed $2 \mathrm{~h}$ later (NS, IS, IS + FLX). The other half of the animals was given the AT (NS/AT, IS/AT, IS + FLX/ AT). No animals were given both the AT and BrdU injections. 
incubated with $2 \mathrm{~N} \mathrm{HCl}$ and blocked with $3 \%$ NHS (Vector Laboratories) and anti-mouse BrdU (1:100; Becton-Dickinson) overnight at $4{ }^{\circ} \mathrm{C}$. They were then incubated for $1 \mathrm{~h}$ with secondary antibody (1:200 biotinylated horse antimouse, Sigma), followed by amplification with an avidinbiotin complex (Vector Laboratories), and cells were visualized with DAB (Pierce). For double-labeling or triple-labeling, sections were incubated for 3 days at $4{ }^{\circ} \mathrm{C}$ with anti-rat $\operatorname{BrdU}(1: 200$; Accurate) and anti-mouse NeuN $(1: 50$; Chemicon) or anti-Rabbit anti-S100 $\beta \quad(1: 2500$; SWant, Switzerland). After washes, Alexa-Fluor secondary antibodies (1:200, Molecular Probes) were applied and visualized with confocal $z$-plane sectioning ( $1 \mu \mathrm{m}$ steps).

A modified unbiased stereology protocol was used that has been reported to successfully quantify BrdU labeling (West et al, 1991; Malberg et al, 2000). Every eighth section throughout the hippocampus was processed for BrdU immunohistochemistry. All BrdU-labeled cells in the dentate gyrus (granule cell layer) and hilus were counted in each section by an experimenter blinded to the study code. To distinguish single cells within clusters, all counts were performed at $\times 400$ and $\times 1000$ magnification under a light microscope, omitting cells in the outermost focal plane. A cell was counted as being in the SGZ of the dentate gyrus if it was touching or in the SGZ. Cells that were located more than two cells away from the SGZ were classified as hilar. The total number of BrdU-labeled cells per slice was determined and multiplied by eight to obtain the total number of cells per dentate gyrus. To confirm that the BrdU was labeling newborn cells and not cells undergoing DNA repair, mitotic figures were observed in each hippocampal slice.

To control for a nonspecific effect of antidepressant treatment on BrdU uptake and labeling and to determine whether the effects are specific to hippocampal neurogenesis, striatal sections were processed for BrdU labeling in the subventricular zone of the lateral ventricle. BrdUpositive cells on the lateral side of the lateral ventricle were counted (10.0-9.0 mm intra-aural; Paxinos and Watson, 1986). At least eight sections were examined per animal and every animal in the group was analyzed. Statistical analysis was performed on the average number of BrdU-positive cells per section. This protocol has been previously used to quantify BrdU labeling in the subventricular zone (Malberg et al, 2000).

For double or triple-labeling, slices were analyzed on a confocal microscope (Zeiss Axiovert LSM510). At least 50 BrdU-positive cells per animal were analyzed using $Z$-plane sectioning ( $1 \mu \mathrm{m}$ steps) to confirm the colocalization of BrdU and the markers NeuN or S100 $\beta$. The percentage of BrdU-positive cells of each phenotype was determined.

\section{Determination of Corticosterone (CORT) Levels}

In a separate experiment, animals were given IS on Day 1 and a postshock test on Day 2 or remained in the box unshocked (NS). Animals were injected with saline on Days $2-8$. On Day 9, blood was drawn from the saphenous vein of awake, nonanesthetized rats. Blood was kept on ice and centrifuged immediately. Plasma was kept at $-20^{\circ} \mathrm{C}$ until analysis. CORT levels were obtained using the Immuno-
Chem Double Antibody Corticosterone RIA kit for rats and mice (ICN Biochemicals, Costa Mesa, CA).

\section{Statistical Analysis}

For learned helplessness, values are expressed as mean escape latency \pm SEM. For proliferation and neurogenesis, values are expressed as the total number of BrdU-labeled cells per section. Experiments with two groups were compared with unpaired Student's $t$-test with a significance level of $P<0.05$. For experiments with three groups, ANOVA and post hoc Tukey tests were performed.

\section{RESULTS}

\section{Exposure to the AT Decreases Cell Proliferation in the Hippocampus}

Experiment 1. In the learned helplessness behavioral paradigm that we used, rats were exposed to a repeated IS on one side of a shuttle box on Day 1. On Day 9, the animals were given a two-way AT, where they had the ability to escape to the unshocked side of the shuttle box. It has been demonstrated that rats previously exposed to IS have greater latencies to escape than control animals who were not previously shocked. This increased latency to escape to the unshocked side is termed behavioral despair or 'learned helplessness' (Willner, 1984; Thiebot et al, 1992; Cryan et al, 2002).

In experiment 1 , rats were given IS (IS group) or were placed in the box only and not shocked (NS group). On Day 2 , a postshock test was performed (see methods). A total of $28 \%$ of IS animals did not meet the criterion and were removed after the postshock test. On Day 9, the AT was performed on the IS and half of the NS groups (referred to as the IS/AT and NS/AT groups). At 5 min after the IS/AT and NS/AT animals were removed from the shuttle box after testing, they were injected with BrdU. The NS group of animals was not exposed to any shock, but was only injected with BrdU on Day 9.

In this experiment, our hypothesis was that the IS/AT rats would have (1) longer latencies to escape than the NS/AT rats, and (2) decreased cell proliferation compared to the NS/AT and NS groups. There was a significant increase in the latency to cross in the IS/AT animals compared to the NS/AT group (NS/AT, $5.6 \pm 1.1 \mathrm{~s} ;$ IS/AT, $26.4 \pm 3.0 \mathrm{~s}$, $P<0.001$ ) (Figure 2a). The short latency in the NS/AT group confirms that, when animals are given the chance to escape a foot-shock, they will quickly learn to escape. The rats in the IS/AT group displayed the expected learned helplessness escape deficit, in agreement with the first part of our hypothesis. However, contrary to the second part of the hypothesis, BrdU cell counts of the IS/AT and NS/AT groups were not different from each other $(P>0.05)$. In addition, the number of BrdU-labeled cells was significantly decreased by approximately $50 \%$ in both the IS/AT and NS/ AT groups compared to the NS group $\left(\mathrm{F}_{(2,24)}=4.3, P<0.05\right.$; Figure $2 b$ ).

These data indicate that exposure to acute shock in the AT decreases cell proliferation by $50 \%$, regardless of escape latency or pretest treatment. Importantly, this experiment shows that the stress associated with the AT itself produces 
a

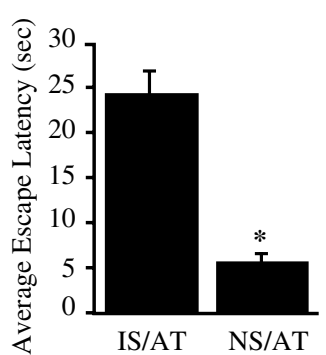

b

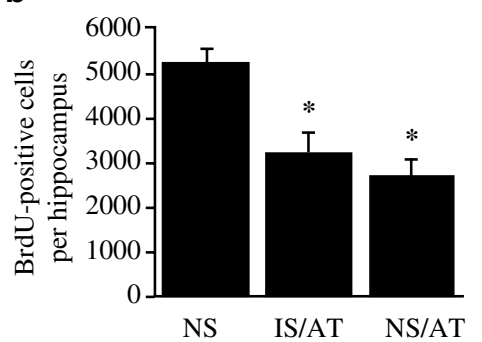

Figure 2 Effect of IS on escape latency and cell proliferation. (a) Animals who were pre-exposed to IS have increased latencies to escape on a subsequent AT compared to animals who had not been previously shocked (NS). Results are expressed as the average escape latency and are the mean \pm SEM in each treatment group ( $n=8$ per group). $* P<0.05$ compared to IS group. (b) NS/AT and IS/AT animals were given BrdU immediately after the AT. The NS group was only given a BrdU injection and was not exposed to either IS or avoidance testing. The IS/AT and NS/ AT groups had a 50\% decrease in BrdU-positive cells compared to the NS group, but were not different from each other. Results are expressed as the number of BrdU-labeled cells and are mean \pm SEM in the total hippocampus per group ( $n=9$ per group). ${ }^{*} P<0.05$ compared to NS group.

acute decreases in proliferation. We conclude that exposure to the AT produces a floor effect, suppressing proliferation to the same extent in both groups. Therefore, using this protocol, it is not possible to determine if the IS/AT and NS/ AT groups had different levels of cell proliferation before the AT. Experiment 2 was designed to separate the effect of IS on Day 1 from the acute effect of the AT on Day 9.

\section{Exposure to IS Results in a Long-Lasting Decrease in Cell Proliferation}

Experiment 2. In this experiment, two changes were made from experiment 1 (see Figure 1 for experimental design): (1) There was an additional treatment group that consisted of animals who received IS and the postshock test, but were injected with fluoxetine (IS + FLX) on Days 2-8. (2) On Day 9, half the animals in each of the three treatment groups (NS, IS, IS + FLX) received injections of BrdU. The other half of the animals in those treatment groups was given the AT (NS/AT, IS/AT, IS + FLX/AT). This protocol was designed as such because we wished to determine the amount of BrdU labeling in the hippocampus of the NS, IS, and IS + FLX rats before the rats were exposed to the AT because of the results obtained in experiment 1 .

For this experiment, 54 rats were given IS on Days 1 and 2. 14 rats $(26 \%)$ did not show escape deficits during the postshock test and were removed from the study. The remaining 40 rats were divided into two groups, IS or IS + FLX. There was also an NS group of 20 rats treated on Days 1 and 2 as described above. On Day 9, half the animals in each of the three groups (NS, IS, and IS + FLX) were injected with BrdU and half of the animals in each treatment group were given the AT (NS/AT, IS/AT, and IS + FLX/AT). In contrast to experiment 1 , none of the animals in experiment 2 were given both the AT and injected with BrdU.

In the AT, the NS/AT group had an average escape latency of $4.8 \pm 0.8 \mathrm{~s}$, compared to $21 \pm 1.5 \mathrm{~s}$ for the IS/AT group

a

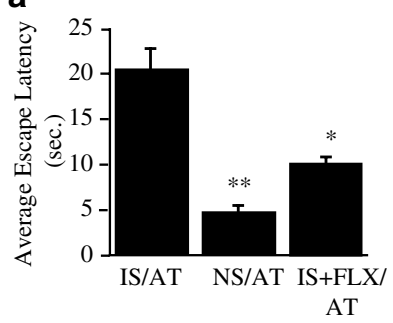

b

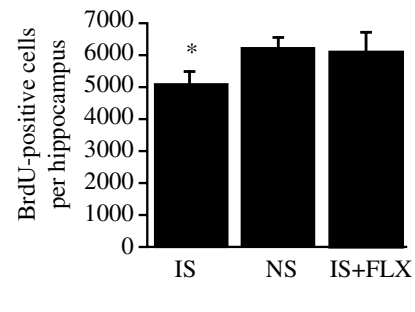

Figure 3 Exposure to IS decreases the number of BrdU-labeled cells in the hippocampus. The paradigm for this experiment is shown in Figure I. Animals were given either the AT or injected with BrdU, but not with both. (a) Animals in the IS/AT group have an escape deficit that is partially and significantly reversed by fluoxetine treatment on Days 2-8 (IS + FLX/AT). Results are expressed as the average escape latency and are the mean \pm SEM in each treatment group ( $n=9$ per group). $* * * 0.01$ compared to IS/AT * $P<0.05$ compared to IS/AT and NS/AT. (b) Animals given IS show a decrease in the number of BrdU-labeled cells compared to NS animals, and this effect is reversed by fluoxetine (IS + FLX) treatment on Days 2-8. Results are expressed as the number of BrdU-labeled cells and are mean \pm SEM in the total hippocampus per group $(n=8$ per group). ${ }^{*} P<0.05$ compared to $N S$ and $I S+F L X$.

and $10 \pm 1.1$ for the IS + FLX/AT (Figure 3a). There was a significant treatment effect between the three groups $\left(\mathrm{F}_{(2,24)}=4.9, P<0.05\right)$. The IS/AT group latencies were significantly higher than the NS/AT group $(P<0.01)$. Although fluoxetine administration did not completely reverse the effect of IS, the latency of the IS + FLX/AT group was significantly different from both the NS/AT and IS/AT shock groups $(P<0.05)$, indicating a significant effect of fluoxetine treatment on learned helplessness behavior.

On Day 9, the other half of the animals from all three groups (IS, IS + FLX, NS) were injected with BrdU (100 mg/ $\mathrm{kg}$ ) and the brains were perfused $2 \mathrm{~h}$ later to investigate the effect of IS and fluoxetine administration on cell proliferation. It is important to note that the animals analyzed for BrdU staining were not exposed to any behavioral tests on Day 9 (see Figure 1), but were analyzed for cell proliferation at the same time as their cage mates were administered the AT. In this way, we were able to quantify a 'baseline' measurement of proliferation at the time of the AT. It can be seen (Figure 3b) that on Day 9, there was a significant effect of treatment on cell proliferation $\left(\mathrm{F}_{(2,21)}=3.8, P<0.05\right)$. The number of BrdU-positive cells in the IS group was significantly lower than both the IS + FLX and NS groups $(P<0.05)$. The IS + FLX and NS groups were not significantly different from each other (Figure $3 \mathrm{~b}$ ). This result demonstrates that when animals are exposed to IS 9 days before analysis of BrdU labeling there is a decrease in cell proliferation, and that this effect is reversed by fluoxetine administration.

To determine whether the effects of IS and IS + FLX on cell proliferation were specific to the hippocampus, cell counts were determined in the subventricular zone of the lateral ventricle, which is another area known to be neurogenic in the adult animal (Alvarez-Buylla and Garcia-Verdugo, 2002). We report that there was no difference $\left(\mathrm{F}_{(2,21)}=1.82, P>0.05\right)$ in cell counts of the three treatment groups (data not shown). This indicates that IS specifically affects cell proliferation in the hippocampus. 
This also provides evidence that among the three treatment groups, there was no change in the amount of BrdU entering the brain or the amount of BrdU incorporated into the DNA of proliferating cells.

\section{Levels of CORT are not Altered 9 Days after IS}

Experiment 3. To determine whether the decrease in proliferation on Day 9 in the IS-treated animals could be due to an increase in CORT, blood levels of this adrenal steroid were analyzed. Animals were exposed to IS on Day 1, and to the postshock test on Day 2. NS animals were exposed to the shuttle box but did not receive foot-shock. In all, $25 \%$ of the IS animals were removed after the postshock test because they did not exhibit an escape latency deficit. On Day 9, blood was drawn from NS and IS animals. Animals were not tested on the AT in this experiment. The detection of CORT levels by radioimmunoassay indicates that IS and NS rats have similar plasma CORT levels (IS, $8.0 \pm 1.3$; NS, $6.9 \pm 1.5 \mu \mathrm{g} / \mathrm{dl}$ ) (Figure 4). This indicates that the decrease in proliferation seen in the IS animals is not due to an increase in CORT levels at the time of killing, and suggests that some other factor accounts for the decrease in cell proliferation resulting from exposure to IS.

\section{Exposure to Escapable Shock does not Produce a Decrease in Cell Proliferation}

Experiment 4. To further test the hypothesis that the decrease in cell proliferation on Day 9 is due to exposure to IS treatment on Day 1 and not merely any exposure to a shock, a separate group of animals was given a two-way AT on Day 1. In this manner, the animals are exposed to ES on Day 1. The ES animals had an average latency to escape of $4.0 \pm 1.0 \mathrm{~s}$. In the postshock test on Day 2, none of the animals in the ES group displayed escape deficits. A NS group remained unshocked in the shuttle box on Days 1 and 2. On Day 9, both ES and NS group animals were analyzed for BrdU labeling. There was no significant difference between the ES and NS groups in the number of BrdUlabeled cells in hippocampus (Figure 5). This finding indicates that, in contrast to the animals given IS, animals

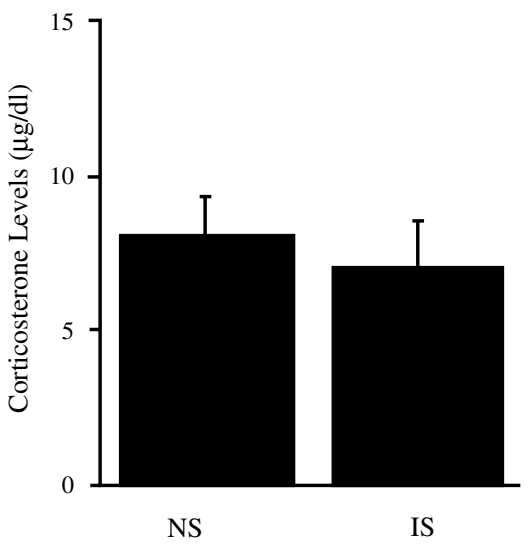

Figure 4 Serum CORT levels do not differ between IS and NS groups. Animals were given IS or NS exposure to the box. On Day 9, serum CORT levels were obtained from awake, nonrestrained animals. Results are given as mean $\pm \mathrm{SEM} \mu \mathrm{g} / \mathrm{dl}$ per group ( $n=8$ per group).

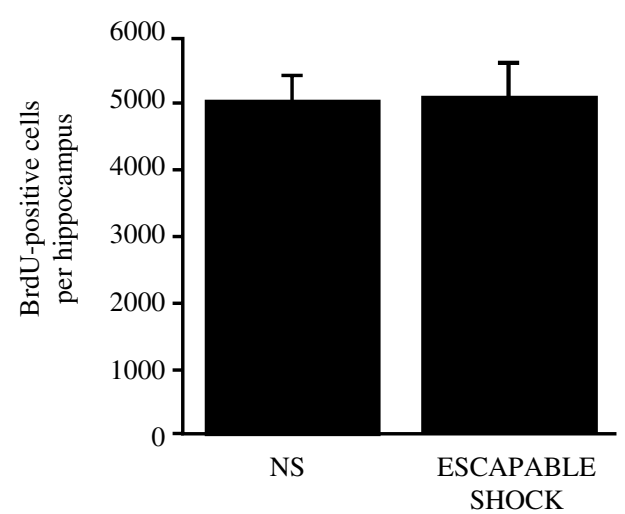

Figure 5 Exposure to ES does not influence the number of BrdUpositive cells. Animals were exposed to escapable foot-shock or NS on Day I and were then injected with BrdU on Day 9 and processed for analysis of labeled cells. Results are expressed as the number of BrdUlabeled cells and are mean \pm SEM in the total hippocampus per group ( $n=8$ per group).

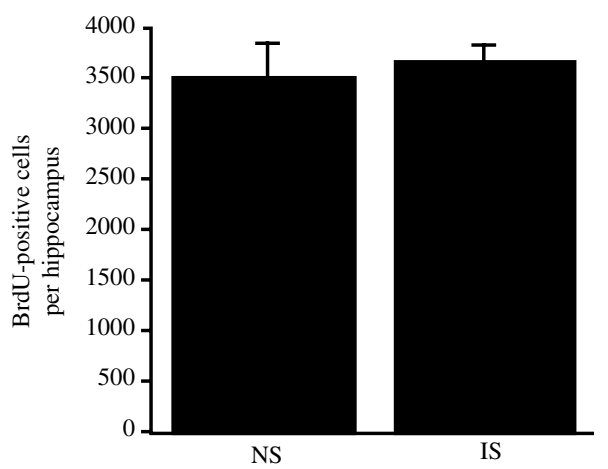

Figure 6 Exposure to IS does not result in decreased BrdU-labeled cells when animals are killed 28 days after injection. Animals were exposed to IS or NS on Day I and then received BrdU injections on Day 9. After 4 weeks, the animals were killed and the number of BrdU-labeled cells was determined. At this time point, there was no significant difference between the group exposed to IS and the NS group. Results are expressed as the number of BrdU-labeled cells and are mean \pm SEM in the total hippocampus per group ( $n=8$ per group).

exposed to a shock from which they can escape do not exhibit baseline changes in cell proliferation 9 days later.

\section{Exposure to IS does not Affect Long-Term Cell Survival and Differentiation}

Experiment 5. To determine whether IS produces long-term effects on the number and phenotype of BrdU-labeled cells, a separate group of animals was given IS or NS exposure on Day 1. After the postshock test on Day 2, 35\% of IS animals were removed. The IS and NS animals were injected with BrdU on Day 9, allowed to survive for 28 days and then killed for BrdU analysis on Day 37. Analysis of BrdUpositive cells revealed that at that time point, there is no difference in the number of BrdU-labeled cells between IS and NS animals $(P>0.05$; Figure 6$)$. This indicates that the decrease in BrdU labeling seen at $2 \mathrm{~h}$ after the injection on Day 9 is not seen 28 days later. A similar result has been observed after exposure to fox odor stress (Tanapat et al, 2001). Triple-labeling of the mature neurons using BrdU, 

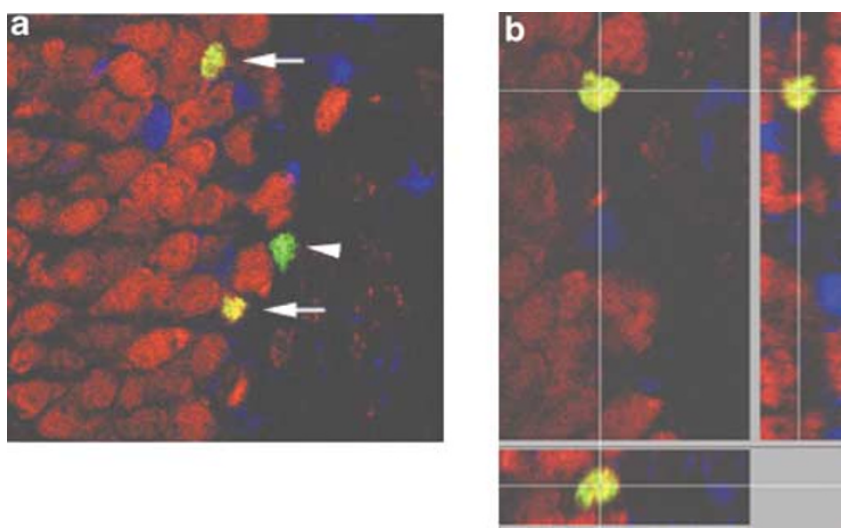

Figure 7 Triple-labeling confirms that the majority of BrdU-positive cells mature into neurons. Sections from the animals analyzed at the 4-week time point were triple-labeled for $\mathrm{BrdU}$ (green), the neuronal marker $\mathrm{NeuN}$ (red), or the glial marker $\mathrm{SI} 00 \beta$ (blue). (a) A representative confocal laser-scanning image from an IS-exposed rat is shown. Arrows indicate cells that are double-labeled for $\mathrm{BrdU}$ and $\mathrm{NeuN}$, while the arrowhead indicates a cell labeled just with BrdU. (b) Confocal image of a cell double-labeled with BrdU and NeuN shown in three planes $(x, y, z)$ to verify doublelabeling throughout its extent.

NeuN, and $S 100 \beta$ (Figure 7) reveals that the percentage of cells double-labeled for neurons and glia did not differ between the IS and NS groups (BrdU $+\mathrm{NeuN}$ : 70\%, BrdU + S100 $\beta$ : $15 \%$; BrdU only: $15 \%$ ). This indicates that the differentiation into neurons and glia was not affected in the IS rats.

\section{DISCUSSION}

In the present series of experiments, we report the novel finding that exposure to IS in the learned helplessness model of depression results in a decrease in cell proliferation in the adult hippocampus. Importantly, we also found that fluoxetine administration reverses the IS-induced decrease in cell proliferation, as well as the behavioral helplessness resulting from exposure to IS. The reversal of both the behavioral deficit and the reduction in cell proliferation by fluoxetine treatment demonstrates a correlation between the regulation of neurogenesis and the action of antidepressant treatment in this behavioral paradigm.

Previous investigators have shown that exposure to psychosocial stressors decreases proliferation and neurogenesis in the adult hippocampus (Gould et al, 1997, 1998; Tanapat et al, 2001). Our results in experiment 1 add to the existing literature by demonstrating that acute exposure to the AT produces a robust $(50 \%)$ decrease in cell proliferation. A similar decrease in proliferation was observed whether the animals were pre-exposed to IS or not. Although the IS/AT animals had greater escape latencies, indicating a greater amount of time that IS/AT animals were exposed to the shock, the cell counts of the IS/AT and NS/ AT groups were not different. This indicates that exposure to the foot-shock during the AT itself results in a floor effect on cell proliferation.

To circumvent the acute effects of avoidance testing, experiment 2 was designed to determine the levels of cell proliferation in animals who were previously exposed to IS, but not subjected to testing just prior to BrdU injection. In this experiment, we found that exposure to IS resulted in a significant downregulation of cell proliferation, determined 9 days after IS. Parallel groups of animals were prepared for behavioral analysis, which demonstrated that IS also resulted in a significant increase in the latency to escape in cage mates of the animals tested for cell proliferation. These results indicate that the behavioral despair resulting from IS exposure is accompanied by decreased cell proliferation in the hippocampus and this effect lasts for at least 9 days. The results from experiments 1 and 2 also indicate that pre-exposure to IS, which resulted in a significant baseline decrease in cell proliferation, does not lead to a greater decrease when animals are also exposed to shock during the avoidance testing.

We also report that fluoxetine administration reverses the downregulation of cell proliferation as well as the behavioral despair resulting from exposure to IS. This finding is in agreement with a recent study reporting that antidepressant treatment reverses the decrease in cell proliferation observed in tree shrews as a result of exposure to intruder stress (Czeh et al, 2001). The latter study tested an atypical antidepressant, tianeptine, for which the acute actions are unknown. The present study extends this finding by demonstrating that fluoxetine, a serotonin selective reuptake inhibitor and one of the most commonly prescribed antidepressants, also blocks IS-induced downregulation of cell proliferation.

The normalization of cell proliferation by 7 days of fluoxetine treatment in this study could result from blockade of the effect of IS, or could reflect an additive action of antidepressant treatment (increase) and IS (decrease) on cell proliferation. In our previous study (Malberg et al, 2000), 14 days of fluoxetine increased cell proliferation by $35-40 \%$ relative to control, whereas 1 day of fluoxetine had no effect and 5 days of fluoxetine produced a nonsignificant $8 \%$ increase. In the current study, we demonstrate that 7 days of fluoxetine increased cell proliferation in IS rats by $20 \%$. Although it would be of interest to have a 7-day NS + fluoxetine group, on the basis of our previous data we hypothesize that 7 days of fluoxetine would not produce the same kind of increase in the NS rats seen in the IS rats.

Our data suggest that the effect of fluoxetine on the IS rats is a specific response to normalize the IS-induced downregulation. However, it is possible that cell proliferation in animals exposed to IS may be more sensitive to antidepressant treatment, resulting in significant upregulation even at this shorter time point. In addition, it may be that the higher dose of fluoxetine used in this study, chosen for its behavioral effects, has a more rapid effect on cell proliferation than the dose used in the previous study. It has been shown that increasing the doses of antidepressants increases synaptic levels of monoamines (Page and Lucki, 2002), and this could result in a greater effect on cell proliferation. Alternatively, it is possible that antidepressant treatment normalizes other neurochemical or cellular responses that are altered by stress and thereby reverses the reduction in cell proliferation as well as the avoidance deficit.

In addition to IS exposure, we felt it was important to examine the influence of ES on cell proliferation 9 days after 
exposure because controllability is a central component of the learned helplessness paradigm (see Maier and Seligman, 1976). In this experiment, the animals were able to escape to the unshocked compartment, and readily did so with a mean latency of approximately $4 \mathrm{~s}$. When these animals were then tested on Day 9, the same time point as the animals exposed to IS, there was no significant difference in cell proliferation. However, there are various interpretations of this result. One argument is that exposure to a foot-shock that is escapable is not sufficient to decrease cell proliferation when analyzed on Day 9, and that it is the inescapable nature of the shock that is required for downregulation of cell proliferation. However, the ES animals received a shorter period of foot-shock ( 30 trials $\times 4 \mathrm{~s})$ than animals exposed to IS (60 trials $\times 15 \mathrm{~s}$ ), and it is possible that the increased levels of stress accounts for the decrease in cell proliferation. Additional studies using a yoked control group that received the same amount of shock as the IS group are needed to further investigate this point.

In this study, $25-35 \%$ of the rats were removed after the postshock test since they were deemed not to be helpless. We did this in order to avoid a false positive behavioral effect, since on Day 9, these animals would show shorter latencies to escape that would be incorrectly attributed to either antidepressant action or spontaneous recovery. However, it may be argued that in fact these animals could serve as an additional control group. Analysis of BrdU labeling in this group would help to determine if the decrease in cell proliferation on Day 9 is dependent on exposure to IS that is accompanied by a behavioral deficit or if only the IS stress is required. Additional studies will be required to test this hypothesis and to establish the molecular determinants that account for this difference.

Stress-induced downregulation of neurogenesis is thought to result from activation of the hypothalamicpituitary-adrenal (HPA) axis and elevated levels of CORT (see McEwen, 1999; Duman et al, 2001). Stress increases serum-bound and free CORT levels, and administration of CORT has been shown to decrease adult neurogenesis (McEwen, 1999). A previous study has demonstrated that exposure to IS produces a large surge in serum CORT levels that lasts for up to $24 \mathrm{~h}$ (Milligan et al, 1998). Although levels of CORT were not determined after avoidance testing in the first experiment, it is likely that levels of the adrenalsteroid are elevated and could account for the decrease in cell proliferation that is observed immediately after avoidance testing (experiment 1). However, 9 days after exposure to IS (experiment 2), we demonstrate that CORT levels are no longer significantly elevated relative to controls, suggesting that the reduction in hippocampal cell proliferation is not due to elevated CORT. Importantly, this finding indicates that exposure to IS produces a condition that is characterized by behavioral helplessness and longterm modulation of cell proliferation.

However, we cannot rule out the possibility that the changes in proliferation observed at the 9-day time point result from elevated CORT during exposure to IS. Elevation of CORT levels during IS, combined with other effects of inescapable stress exposure, could result in a condition where the animals become hyper-responsive to subsequent injections or handling. This hyper-responsiveness could then lead to increased CORT after BrdU injection and produce the decrease in proliferation on Day 9. In support of this, Bruijnzeel et al (2001) have demonstrated that animals exposed to a single session of foot-shocks had no baseline change in CORT or ACTH, but exhibited increased stress responsivity when tested 2 weeks later.

Previous studies have demonstrated that physiologically relevant stressors, including psychosocial stress, intruder stress, and predator odor (Gould et al, 1997, 1998; Tanapat et al, 1998; Czeh et al, 2001) decrease hippocampal proliferation. Although in the present study we use a nonphysiological stressor, we report that the magnitude of decrease in cell proliferation that we observe with footshock is similar to that observed in the previous studies. In addition, we demonstrate that there is no significant effect of IS on the number of BrdU-labeled cells 28 days after BrdU injection, which is consistent with a previous report on fox odor stress (Tanapat et al, 2001). Moreover, there are no reports in the literature demonstrating that the downregulation of cell proliferation resulting from acute stress translates into a decrease in the number of mature neurons determined at a later time point. Taken together, these findings add to the existing findings on the effects of IS and stress on the brain (McEwen, 2001; Nestler et al, 2002; Stewart and Reid, 2002) and indicates a complex effect of stress on neurogenesis at the longer time points examined.

The explanation for the lack of effect of stress on neurogenesis at the later time point has not been determined, but it could be related to alterations in the survival of cells. It has been shown that approximately $50 \%$ of newly labeled BrdU-positive cells in the hippocampus do not survive longer than 2 or 3 weeks (Hastings and Gould, 1999). Given the acute decrease in cell proliferation that is seen, our results indicate that there may be a compensatory response in the IS-exposed animals that results in increased survival of BrdU-labeled cells relative to unstressed controls. This type of compensatory mechanism could have evolved to maintain the appropriate number of new neurons, even after exposure to acute stress. Alternatively, the control animals may be overproducing new cells. We have demonstrated that the IS animals are producing fewer cells, so there may be less cell death or less competition for trophic support in these animals. In this way, the proportion of cells that survive would be lower in the control group than the IS group, but the net number of surviving cells would be the same as we observed.

It is interesting to note that the lack of effect of IS on cell survival correlates with the loss of the behavioral effects of IS over time. It is well known that rodents recover from the behavioral despair by 2-3 weeks after exposure to IS (see Cryan et al, 2002). It is possible that this behavioral recovery is associated with the increased survival of BrdUlabeled cells. Additional studies will be required to directly determine if there is in fact an increase in the survival of newborn cells after exposure to IS and other stressors. It has been shown that repeated exposure to an aversive stimulus prolongs behavioral deficits in the learned helplessness model (Maier, 2001), and it is possible that such a paradigm would also result in decreased numbers of BrdU-labeled cells determined at the later time point.

Although the exact mechanisms underlying the regulation of neurogenesis by IS stress and antidepressant treatment have not been determined, there are several possibilities. 
Downregulation of neurogenesis by glucocorticoids is blocked by NMDA receptor antagonists (Cameron et al, 1998), and it is possible that activation of NMDA receptors could account for the decrease caused by IS. Adult neurogenesis is dependent on an intact 5-HT system (Brezun and Daszuta, 1999) and it is possible that disruption of this monoamine system accounts for the downregulation of neurogenesis, as well as the blockade of this effect by fluoxetine treatment. Yet another possibility is brain-derived neurotrophic factor (BDNF), which is downregulated by stress (Nibuya et al, 1995; Smith et al, 1995) and is reported to increase neurogenesis (Pencea et al, 2001; Lee et al, 2002). We have found that antidepressant treatment increases BDNF and blocks the downregulation of this neurotrophic factor by stress (Nibuya et al, 1995), consistent with the effects of antidepressant treatment on cell proliferation. Further studies will be needed to determine which of these neurotransmitters or factors can account for the regulation of cell proliferation by IS and antidepressant treatment.

In summary, these experiments show that acute exposure to an AT, a stress paradigm that activates the HPA axis, acutely decreases cell proliferation in the adult hippocampus. Moreover, the results demonstrate that prior exposure to IS, in the learned helplessness model of depression, produces a decrease in cell proliferation after the acute effects of stress on the HPA axis have dissipated. We also report that antidepressant treatment reverses the effect of IS on cell proliferation, as well as the behavioral deficits resulting from IS exposure. Further studies will be necessary to delineate the interaction between animal models of depression, cell proliferation and neurogenesis, and the mechanism of antidepressant action.

\section{ACKNOWLEDGEMENTS}

This work was supported by USPHS Grants MH45481 and 2 PO1 MH25642, a Veterans Administration National Center Grant for PTSD, and by the Connecticut Mental Health Center.

\section{REFERENCES}

Alvarez-Buylla A, Garcia-Verdugo JM (2002). Neurogenesis in adult subventricular zone. J Neurosci 22: 629-634.

Bremner JD, Narayan M, Anderson ER, Staib LH, Miller HL, Charney DS (2000). Hippocampal volume reduction in major depression. Am J Psychiatry 157: 115-118.

Bremner JD, Randall P, Scott TM, Bronen RA, Seibyl JP, Southwick SM et al (1995). MRI-based measurement of hippocampal volume in patients with combat-related posttraumatic stress disorder. Am J Psychiatry 152: 973-981.

Brezun JM, Daszuta A (1999). Depletion in serotonin decreases neurogenesis in the dentate gyrus and the subventricular zone of adult rats. Neuroscience 89: 999-1002.

Bruijnzeel AW, Stam R, Compaan JC, Wiegant VM (2001). Stress-induced sensitization of CRH-ir but not P-CREB-ir responsivity in the rat central nervous system. Brain Res 908: 187-196.

Cameron HA, McKay RD (2001). Adult neurogenesis produces a large pool of new granule cells in the dentate gyrus. J Comp Neurol 435: 406-417.
Cameron HA, Tanapat P, Gould E (1998). Adrenal steroids and $\mathrm{N}$ methyl-D-aspartate receptor activation regulate neurogenesis in the dentate gyrus of adult rats through a common pathway. Neuroscience 82: 349-354.

Cryan JF, Markou A, Lucki I (2002). Assessing antidepressant activity in rodents: recent developments and future needs. Trends Pharmacol Sci 23: 238-245.

Czeh B, Michaelis T, Watanabe T, Frahm J, de Biurrun G, van Kampen $M$ et al (2001). Stress-induced changes in cerebral metabolites, hippocampal volume, and cell proliferation are prevented by antidepressant treatment with tianeptine. Proc Natl Acad Sci USA 98: 12796-12801.

Duman RS, Malberg J, Nakagawa S (2001). Regulation of adult neurogenesis by psychotropic drugs and stress. J Pharmacol Exp Ther 299: 401-407.

Gage FH (2000). Mammalian neural stem cells. Science 287: 14331438.

Gilbertson MW, Shenton ME, Ciszewski A, Kasai K, Lasko NB, Orr SP et al (2002). Smaller hippocampal volume predicts pathologic vulnerability to psychological trauma. Nat Neurosci 5: $1242-$ 1247.

Gould E, McEwen BS, Tanapat P, Galea LA, Fuchs E (1997). Neurogenesis in the dentate gyrus of the adult tree shrew is regulated by psychosocial stress and NMDA receptor activation. J Neurosci 17: 2492-2498.

Gould E, Tanapat P, McEwen BS, Flugge G, Fuchs E (1998). Proliferation of granule cell precursors in the dentate gyrus of adult monkeys is diminished by stress. Proc Natl Acad Sci USA 95: $3168-3171$.

Gould E, Tanapat P, Rydel T, Hastings N (2000). Regulation of hippocampal neurogenesis in adulthood. Biol Psychiatry 48: 715-720.

Hastings NB, Gould E (1999). Rapid extension of axons into the CA3 region by adult-generated granule cells. J Comp Neurol 413: 146-154.

Kempermann G (2002). Why new neurons? Possible functions for adult hippocampal neurogenesis. J Neurosci 22: 635-638.

Lee J, Duan W, Mattson MP (2002). Evidence that brain-derived neurotrophic factor is required for basal neurogenesis and mediates, in part, the enhancement of neurogenesis by dietary restriction in the hippocampus of adult mice. J Neurochem 82: $1367-1375$.

Madsen TM, Treschow A, Bengzon J, Bolwig TG, Lindvall O, Tingstrom A (2000). Increased neurogenesis in a model of electroconvulsive therapy. Biol Psychiatry 47: 1043-1049.

Magarinos AM, McEwen BS, Flugge G, Fuchs E (1996). Chronic psychosocial stress causes apical dendritic atrophy of hippocampal CA3 pyramidal neurons in subordinate tree shrews. $J$ Neurosci 16: 3534-3540.

Maier SF (2001). Exposure to the stressor environment prevents the temporal dissipation of behavioral depression/learned helplessness. Biol Psychiatry 49: 763-773.

Maier SF, Seligman MEP (1976). Learned helplessness: theory and evidence. J Exp Psychol 105: 3-46.

Malberg JE, Eisch AJ, Nestler EJ, Duman RS (2000). Chronic antidepressant treatment increases neurogenesis in adult rat hippocampus. J Neurosci 20: 9104-9110.

Manev H, Uz T, Smalheiser NR, Manev R (2001). Antidepressants alter cell proliferation in the adult brain in vivo and in neural cultures in vitro. Eur J Pharmacol 411: 67-70.

McEwen BS (1999). Stress and hippocampal plasticity. Annu Rev Neurosci 22: 105-122.

McEwen BS (2001). Plasticity of the hippocampus: adaptation to chronic stress and allostatic load. Ann NY Acad Sci 933: 265-277.

Milligan ED, Nguyen KT, Deak T, Hinde JL, Fleshner M, Watkins LR et al (1998). The long term acute phase-like responses that follow acute stressor exposure are blocked by alpha-melanocyte stimulating hormone. Brain Res 810: 48-58. 
Nestler EJ, Barrot M, DiLeone RJ, Eisch AJ, Gold SJ, Monteggia LM (2002). Neurobiology of depression. Neuron 34: 13-25.

Nibuya M, Morinobu S, Duman RS (1995). Regulation of BDNF and trkB mRNA in rat brain by chronic electroconvulsive seizure and antidepressant drug treatments. J Neurosci 15: 7539-7547.

Page ME, Lucki I (2002). Effects of acute and chronic reboxetine treatment on stress-induced monoamine efflux in the rat frontal cortex. Neuropsychopharmacology 27: 237-247.

Paxinos G, Watson C (1986). The Rat Brain in Stereotaxic Coordinates. Academic Press: San Diego.

Pencea V, Bingaman KD, Wiegand SJ, Luskin MB (2001). Infusion of brain-derived neurotrophic factor into the lateral ventricle of the adult rat leads to new neurons in the parenchyma of the striatum, septum, thalamus, and hypothalamus. J Neurosci 21: 6706-6717.

Porsolt RD (2000). Animal models of depression: utility for transgenic research. Rev Neurosci 11: 53-58.

Sheline YI, Sanghavi M, Mintun MA, Gado MH (1999). Depression duration but not age predicts hippocampal volume loss in medically healthy women with recurrent major depression. $J$ Neurosci 19: 5034-5043.

Sheline YI, Wang PW, Gado MH, Csernansky JG, Vannier MW (1996). Hippocampal atrophy in recurrent major depression. Proc Natl Acad Sci USA 93: 3908-3913.

Shirayama Y, Chen AC, Nakagawa S, Russell DS, Duman RS (2002). Brain-derived neurotrophic factor produces antidepressant effects in behavioral models of depression. J Neurosci 22: 3251-3261.

Smith MA, Makino S, Kvetnansky R, Post RM (1995). Stress and glucocorticoids affect the expression of brain-derived neurotrophic factor and neurotrophin-3 mRNAs in the hippocampus. J Neurosci 15: 1768-1777.
Stewart CA, Reid IC (2002). Antidepressant mechanisms: functional and molecular correlates of excitatory amino acid neurotransmission. Mol Psychiatry 7: S15-S22.

Takahashi T, Nowakowski RS, Caviness VS (1992). BUdR as an Sphase marker for quantitative studies of cytokinetic behaviour in the murine cerebral ventricular zone. J Neurocytol 21: 185-197.

Tanapat P, Galea LA, Gould E (1998). Stress inhibits the proliferation of granule cell precursors in the developing dentate gyrus. Int J Dev Neurosci 16: 235-239.

Tanapat P, Hastings NB, Rydel TA, Galea LA, Gould E (2001). Exposure to fox odor inhibits cell proliferation in the hippocampus of adult rats via an adrenal hormone-dependent mechanism. J Comp Neurol 437: 496-504.

Thiebot MH, Martin P, Puech AJ (1992). Animal behavioural studies in the evaluation of antidepressant drugs. Br J Psychiatry 15(Suppl): 44-50.

van Praag H, Schinder AF, Christie BR, Toni N, Palmer TD, Gage FH (2002). Functional neurogenesis in the adult hippocampus. Nature 415: 1030-1034.

Vollmayr B, Henn FA. (2001). Learned helplessness in the rat: improvements in validity and reliability. Brain Res Brain Res Protoc 8: 1-7.

Watanabe Y, Gould E, Daniels DC, Cameron H, McEwen BS (1992). Tianeptine attenuates stress-induced morphological changes in the hippocampus. Eur J Pharmacol 222: 157-162.

West MJ, Slomianka L, Gundersen HJ (1991). Unbiased stereological estimation of the total number of neurons in the subdivisions of the rat hippocampus using the optical fractionator. Anat Rec 231: 482-497.

Willner P (1984). The validity of animal models of depression. Psychopharmacology (Berl) 83: 1-16. 